\title{
Identification modale d'un satellite en campagne de qualification
}

\author{
Alain GiRARD ${ }^{\mathrm{a}}$ \\ INTESPACE, 2 rond-point Pierre Guillaumat, BP 64356, 31029 Toulouse Cedex 4, France
}

Reçu 9 décembre 2004, accepté le 21 mars 2005

\begin{abstract}
Résumé - La qualification des satellites se fait entre autres par des essais de type sinus balayé sur vibrateur, d'où l'idée de profiter de ces essais pour identifier les modes propres, au lieu de faire séparément une analyse modale classique, afin de gagner en coûts et délais. Cela nécessite de considérer une excitation du spécimen par sa base et non par force, et d'exploiter très rapidement les données d'essai. Cet article propose une solution générale basée sur le concept de paramètres modaux effectifs. Le traitement, compatible avec une session interactive, privilégie la simplicité d'utilisation et la robustesse, éventuellement au détriment d'une certaine précision. La méthodologie est présentée et illustrée par un exemple industriel dans le contexte d'une étude pour l'Agence Spatiale Européenne.
\end{abstract}

Mots clés : Identification modale / modes propres / essais de vibration / essais sur vibrateurs / essai sinus balayé / satellites

\begin{abstract}
Modal identification of a satellite during qualification campaign. The qualification of satellites includes sine sweep tests on shaker tables which can be also used to identify the eigenmodes, instead of performing separately a standard modal survey test, for reduction of cost and development time. This requires to consider a base driven excitation for the specimen instead of force excitation, and to analyse rapidly the test results. A general solution based on modal effective parameter concept is proposed. The processing, compatible with an interactive session, favours simplicity of use and robustness, possibly to the detriment of some accuracy. The methodology is presented and illustrated with an industrial example in the context of a study for the European Space Agency.
\end{abstract}

Key words: Modal survey test / eigenmodes / vibration tests / shaker test / sine sweep test / satellites

\section{Introduction}

L'identification modale fait en général l'objet d'un essai spécifique à l'aide de petits vibrateurs fournissant des accélérances (rapports accélération/force dans le domaine des fréquences) qui restitueront les propriétés modales par identification de paramètres. L'idée émise dans le domaine spatial, où la qualification des satellites se fait entre autres par des essais de type sinus balayé sur vibrateur, est de profiter de ces essais pour identifier les modes propres dans des configurations plus proches de celles en service (satellite sur lanceur), afin de gagner en coûts et délais. Cela nécessite de considérer une excitation du spécimen par sa base (interface avec le lanceur), ce qui est différent d'une excitation par forces, et d'exploiter les données d'essai en temps quasi-réel, c'est-à-dire entre deux balayages sinus, afin de ne pas allonger la durée de la campagne et de maximiser le retour d'expérience.

\footnotetext{
a Auteur correspondant : alain.girard@intespace.fr
}

Cet article présente une solution applicable dans le cas général d'une structure excitée par forces ou par la base, avec une approche simplifiée pour l'extraction des paramètres modaux. Cette approche est basée sur le concept de paramètres modaux effectifs.

\section{Paramètres modaux effectifs}

Le concept de paramètres modaux effectifs a été introduit à partir des années 70 pour répondre à un certain nombre de questions soulevées dans les calculs de réponse par superposition modale. Sont d'abord apparues les masses effectives qui ont rapidement fait l'objet d'un usage intensif, en particulier dans le domaine aérospatial, puis d'autres paramètres modaux ont été mis en évidence dans une approche unifiée de ces paramètres. Leur utilisation en dynamique des structures est maintenant généralisée, permettant la détection et la sélection des modes importants relatifs à une réponse 


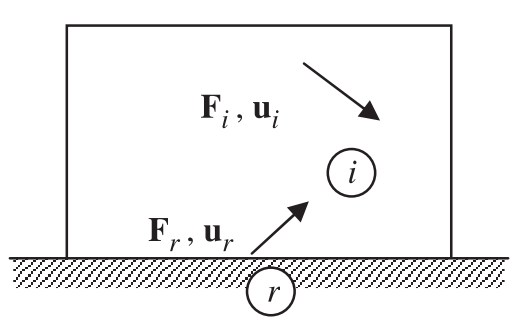

Fig. 1. Forces et déplacements.

donnée, l'évaluation et les effets de troncature modale, la comparaison de bases modales issues d'analyses et/ou d'essais, l'élaboration de modèles équivalents et le calcul explicite des réponses de toutes natures [1].

Dans le contexte de l'identification modale, qui est le problème inverse de la superposition modale, les paramètres modaux effectifs peuvent être utilisés efficacement en raison de leur signification physique liée à une intervention directe dans les fonctions de transfert mesurées.

Le cas général suppose la structure excitée en force sur un certain nombre de DDL (Degrés De Liberté) et en mouvement sur d'autres. On considèrera ici uniquement le cas pratique du mouvement d'une base rigide, même si l'extension au cas général ne pose aucun problème de principe. On a alors pour la structure la partition de DDL suivante :

- les DDL de la base rigide, constituant l'ensemble $r$, avec 6 composantes dans le cas général, où l'on peut imposer des mouvements et récupérer des forces de réaction,

- les autres DDL, qualifiés d'internes et constituant l'ensemble $i$, avec $N$ composantes, où l'on peut imposer des forces et récupérer des mouvements.

On adoptera pour la suite la convention suivante : $\mathbf{X}_{a b}$ désigne une matrice de dimension $(n, p)$ dont les lignes sont relatives aux $n$ DDL $a$ et les colonnes aux $p$ DDL $b$. Ceci implique la relation $\mathbf{X}_{b a}=\mathbf{X}_{a b}$ transposée, et le fait que $\mathbf{X}_{a a}$ soit symétrique, ce qui est licite dans le présent contexte tant que s'applique le principe de réciprocité. De plus, un indice souligné a une valeur fixée : ainsi, $\mathbf{X}_{\underline{a} b}$ par exemple désigne la ligne $\underline{a}$ de $\mathbf{X}_{a b}$.

L'excitation est alors constituée des mouvements imposés, représentés par exemple par les déplacements, d'où le vecteur $\boldsymbol{u}_{r}$, et des forces appliquées, d'où le vecteur $\boldsymbol{F}_{i}$ (Fig. 1). Dans le domaine des pulsations $\omega=2 \pi f$, la relation entre les excitations possibles $\boldsymbol{F}_{i}, \boldsymbol{u}_{r}$, et les réponses $\boldsymbol{u}_{i}, \boldsymbol{F}_{r}$, s'écrit à l'aide des fonctions de transfert:

$$
\left[\begin{array}{l}
\boldsymbol{u}_{i}(\omega) \\
\boldsymbol{F}_{r}(\omega)
\end{array}\right]=\left[\begin{array}{ll}
\mathbf{G}_{i i}(\omega) & \mathbf{T}_{i r}(\omega) \\
-\mathbf{T}_{r i}(\omega) & \mathbf{K}_{r r}(\omega)
\end{array}\right]\left[\begin{array}{l}
\boldsymbol{F}_{i}(\omega) \\
\boldsymbol{u}_{r}(\omega)
\end{array}\right]
$$

où $\mathbf{G}, \mathbf{T}$ et $\mathbf{K}$ sont les matrices de transfert en flexibilité, transmissibilité et raideur dynamiques respectivement.

La superposition modale avec les modes propres $k$ conduit aux expressions suivantes pour les flexibilités $\mathbf{G}$, les transmissibilités $\mathbf{T}$ et les masses (forces/accélérations) $\mathbf{M}=\mathbf{K} /\left(-\omega^{2}\right)[1]$ :

$$
\begin{aligned}
\mathbf{G}_{i i}(\omega) & \approx \sum_{\underline{k}} H_{\underline{k}}(\omega) \tilde{\mathbf{G}}_{i i, \underline{k}}+\mathbf{G}_{i i, \mathrm{res}} \\
\mathbf{T}_{i r}(\omega) & \approx \sum_{\underline{k}} T_{\underline{k}}(\omega) \tilde{\mathbf{T}}_{i r, \underline{k}}+\mathbf{T}_{i r, \mathrm{res}} \\
\mathbf{M}_{r r}(\omega) & \approx \sum_{\underline{k}} T_{\underline{k}}(\omega) \tilde{\mathbf{M}}_{r r, \underline{k}}+\mathbf{M}_{r r, \mathrm{res}}
\end{aligned}
$$

où $H_{\underline{k}}$ et $T_{\underline{k}}$ sont les facteurs sans dimension d'amplification et de transmissibilité dynamique du mode $\underline{k}$ respectivement, fonctions de sa pulsation $\omega_{\underline{k}}$ et de son amortissement visqueux réduit $\zeta_{\underline{k}}$ :

$$
\begin{aligned}
& H_{\underline{k}}(\omega)=\frac{1}{1-\left(\frac{\omega}{\omega_{\underline{k}}}\right)^{2}+i 2 \zeta_{\underline{k}} \frac{\omega}{\omega_{\underline{k}}}} \\
& T_{\underline{k}}(\omega)=\frac{1+i 2 \zeta_{\underline{k}} \frac{\omega}{\omega_{\underline{k}}}}{1-\left(\frac{\omega}{\omega_{\underline{k}}}\right)^{2}+i 2 \zeta_{\underline{k}} \frac{\omega}{\omega_{\underline{k}}}}
\end{aligned}
$$

$\tilde{\mathbf{G}}_{i i, \underline{k}}, \tilde{\mathbf{T}}_{i r, \underline{k}}$ et $\tilde{\mathbf{M}}_{r r, \underline{k}}$ sont les matrices de paramètres effectifs en flexibilité, transmissibilité et masse du mode $\underline{k}$ respectivement, reliées aux modes propres $\boldsymbol{\Phi}_{i k}$, aux facteurs de participation modaux $\mathbf{L}_{\underline{k} r}$ et aux masses généralisées $m_{\underline{k}}$ par :

$$
\tilde{\mathbf{G}}_{i i, \underline{k}}=\frac{\boldsymbol{\Phi}_{i \underline{k}} \underline{\boldsymbol{\Phi}}_{\underline{k} i}}{\omega_{\underline{k}}^{2} m_{\underline{k}}} \quad \tilde{\mathbf{T}}_{i r, \underline{k}}=\frac{\boldsymbol{\Phi}_{i \underline{k}} \mathbf{L}_{\underline{k} r}}{m_{\underline{k}}} \quad \tilde{\mathbf{M}}_{r r, \underline{k}}=\frac{\mathbf{L}_{r \underline{k}} \mathbf{L}_{\underline{k} r}}{m_{\underline{k}}}
$$

$\mathbf{G}_{i i \text {,res }}, \mathbf{T}_{i r \text {,res }}$ et $\mathbf{M}_{r r \text {,res sont les matrices résiduelles }}$ en flexibilité, transmissibilité et masse respectivement, représentant les modes supérieurs et permettant d'être statiquement corrects (statique $=$ dynamique à $\omega=0$ ) :

$$
\begin{aligned}
\sum_{\underline{k}} \tilde{\mathbf{G}}_{i i, \underline{k}}+\mathbf{G}_{i i, \mathrm{res}} & =\mathbf{G}_{i i} \\
\sum_{\underline{k}} \tilde{\mathbf{T}}_{i r, \underline{k}}+\mathbf{T}_{i r, \mathrm{res}} & =\mathbf{\Psi}_{\mathbf{i r}} \\
\sum_{\underline{k}} \tilde{\mathbf{M}}_{r r, \underline{k}}+\mathbf{M}_{r r, \mathrm{res}} & =\overline{\mathbf{M}}_{r r}
\end{aligned}
$$

où $\mathbf{G}_{i i}, \boldsymbol{\Psi}_{i r}$ et $\overline{\mathbf{M}}_{r r}$ sont les matrices de flexibilité (pseudoflexibilité pour les structures libres) statique, de modes rigides et de masse de corps rigide respectivement.

Ainsi, d'après les équations (2)-(4) qui introduisent la notion de paramètre effectif, la contribution de chaque mode propre à un transfert donné est le produit d'un facteur d'amplification sans dimension, fonction de la pulsation propre et de l'amortissement réduit, par le paramètre effectif correspondant. Ce paramètre effectif est indépendant de la normalisation du mode, a la même dimension que le transfert et est directement lié au paramètre statique correspondant. Il mesure de ce fait l'importance du mode pour le transfert considéré, 
indépendamment de l'amplification dynamique, d'où le qualificatif de « effectif » pour souligner cette propriété. Ces paramètres modaux doivent être complétés par des termes résiduels, liés aux paramètres statiques par les relations (7), pour fournir une correction au premier ordre des effets de la troncature modale.

\section{Identification modale}

Les considérations précédentes montrent que l'identification modale des fonctions de transfert mesurées peut se réduire à la détermination de trois types de paramètres sans perte d'information : pulsations propres, amortissements visqueux réduits et paramètres modaux effectifs. Les paramètres modaux habituels comme les formes propres et les masses généralisées peuvent être déduits des paramètres effectifs par les équations (6) si on le souhaite.

L'équation (2) sera utilisée dans le contexte des essais modaux classiques où les excitations par forces et les réponses accélérométriques fournissent les accélérances, c'est-à-dire les flexibilités dynamiques multipliées par $-\omega^{2}$. Les équations (3) et (4) seront utilisées pour une excitation par la base fournissant les transmissibilités dynamiques, ainsi que les masses dynamiques si les réactions à la base sont mesurées. D'une manière générale, avec un seul DDL d'excitation $\underline{e}$ et un ensemble de réponses actives $a$, ces transferts $\mathbf{X}$ (de type $\mathbf{G}$, $\mathbf{T}$ ou $\mathbf{M}$ ) peuvent se mettre sous la forme générale, comme illustré par la figure 2 :

$$
\mathbf{X}_{a \underline{e}}(\omega) \approx\left(\frac{\mathbf{X}_{a \underline{e}, \mathrm{in}}}{-\omega^{2}}\right)+\mathbf{X}_{a \underline{e}, \mathrm{st}}+\sum_{\underline{k}} A_{\underline{k}}(\omega) \tilde{\mathbf{X}}_{a \underline{e}, \underline{k}}
$$

avec :

$\mathbf{X}_{a e, \text { in }}$ contributions inertielles pour une structure libre (modes rigides ou modes $\underline{k}$ inférieurs par rapport à la bande de fréquence considérée, comme les modes de suspension) données par $\boldsymbol{\Phi}_{a \underline{k}} \boldsymbol{\Phi}_{\underline{k e}} / m_{\underline{k}}$

$\mathbf{X}_{a e, s t}$ termes statiques $\left(\mathbf{X}_{a \underline{e}}(0)\right.$ pour une structure non libre) $\mathbf{G}_{a \underline{e}}, \boldsymbol{\Psi}_{a \underline{e}}$ ou $\overline{\mathbf{M}}_{a \underline{e}}$

$\tilde{\mathbf{X}}_{a \underline{e}, \underline{k}}$ paramètres modaux effectifs donnés par les équations (6) et associés aux facteurs d'amplification $A_{k}(\omega)$ dérivés des équations (5) par : $\left(H_{\underline{k}}(\omega)-\overline{1}\right)$ ou $\left(T_{\underline{k}}(\omega)-1\right)$

L'identification de paramètres d'après l'équation (8) fournit :

- les paramètres statiques $\mathbf{X}_{a e, s t}$ et $\mathbf{X}_{a e, \text { in }}$ si présents, d'après les résultats aux basses fréquences,

- les paramètres modaux $\omega_{\underline{k}}, \underline{\zeta_{k}}$ et $\tilde{\mathbf{X}}_{a \underline{e}, \underline{k}}$, d'après les pics : schématiquement, si le mode $\underline{k}$ est bien isolé, $\omega_{\underline{k}}$ est la fréquence des pics, $\zeta_{\underline{k}}$ leur acuité et $\tilde{\mathbf{X}}_{a \underline{e}, \underline{k}}$ se déduit de l'amplitude des pics. Bien entendu, une identification plus précise est à faire avec l'équation (8).

$\mathrm{Si}$ on le souhaite, les formes propres $\boldsymbol{\Phi}_{i \underline{k}}$, les masses généralisées $m_{k}$ et les facteurs de participation $\mathbf{L}_{r \underline{k}}$ peuvent être restitués à partir des $\tilde{\mathbf{X}}_{a \underline{e}, \underline{k}}$ par les

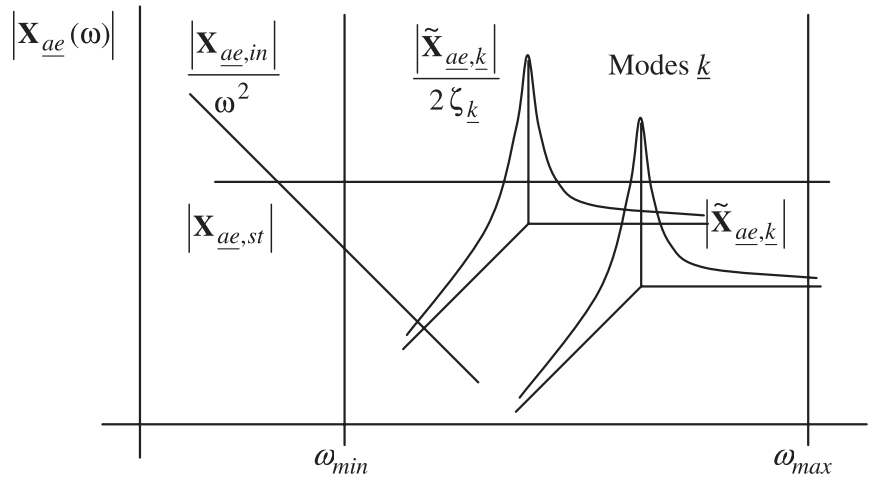

Fig. 2. Décomposition des fonctions de transfert.

équations (6) particularisées aux DDL $a$ et $\underline{e}$. On peut voir sur ces équations que $\tilde{\mathbf{X}}_{\underline{e e}, \underline{k}}$, provenant du transfert ponctuel $\mathbf{X}_{\underline{e e}}(\omega)$, c'est-à-dire $\overline{\mathbf{G}}_{\underline{e e}}(\omega)$ ou $\mathbf{M}_{\underline{e e}}(\omega)$, joue un rôle particulier. Si $m_{k}$ est normalisé à 1, l'équation (6a) fournit la composante ponctuelle $\Phi_{e k}$ de $\tilde{\mathbf{G}}_{e e, k}$ (excitation par force), l'équation (6c) la composante ponctuelle $\mathbf{L}_{\underline{e k}}$ de $\tilde{\mathbf{M}}_{\underline{e e}, \underline{k}}$ (excitation par mouvement). Ensuite, les autres composantes sont déduites des autres $\tilde{\mathbf{X}}_{a \underline{e}, \underline{k}}$.

En fait, le transfert ponctuel $\mathbf{X}_{\underline{e e}}(\omega)$ n'est pas disponible avec une excitation par la base si la réaction n'est pas mesurée, contrairement à l'essai modal classique où la force injectée est en général connue. Dans ce cas, seules les transmissibilités sont déterminées et la composante ponctuelle $\mathbf{L}_{\underline{e k}}$ ne peut être identifiée, à moins de lui donner une valeur arbitraire, mais avec une masse généralisée $m_{\underline{k}}$ indéterminée. Par contre, les autres paramètres sont accessibles : $\omega_{\underline{k}}, \zeta_{\underline{k}}$ et $\tilde{\mathbf{T}}_{a \underline{e}, \underline{k}}$ dont on déduira les formes propres non-normalisées $\bar{\Phi}_{a k}$. Sans mesure de réaction, l'identification est donc possible mais incomplète.

D'après la forme de l'équation (8), la partie imaginaire des transferts n'est due qu'aux modes propres et il est donc préférable d'identifier les paramètres modaux d'après les parties imaginaires plutôt que les amplitudes, comme illustré par la figure 3, avec les avantages suivants : présence du signe, fréquence du pic plus proche de la fréquence propre, influence des modes voisins plus faible, et possibilité d'extraction des paramètres par algorithme linéaire. Après l'identification des paramètres modaux, les paramètres statiques seront déterminés à partir des parties réelles aux basses fréquences.

Dans le contexte d'une campagne d'essais de qualification qui demande d'exploiter très rapidement les données pour obtenir les modes propres les plus importants, priorité doit être donnée à la simplicité d'utilisation de l'outil et à sa robustesse, éventuellement au détriment d'une certaine précision. Une approche compatible avec une session interactive a été proposée dans le contexte d'une étude pour l'Agence Spatiale Européenne [2]. En résumé :

- Visualisation des parties réelles et imaginaires de l'ensemble des accélérations et forces mesurées pour la détection/sélection des modes propres. 

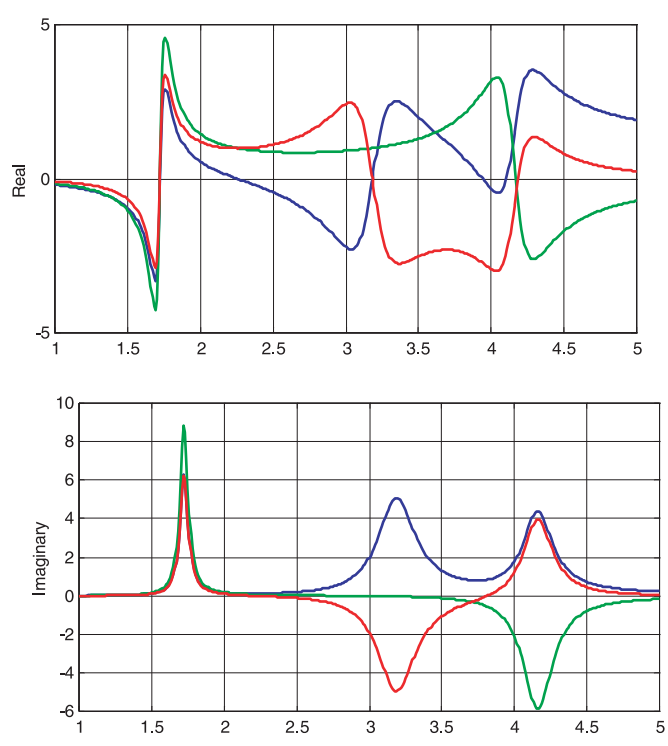

Réel/imaginaire
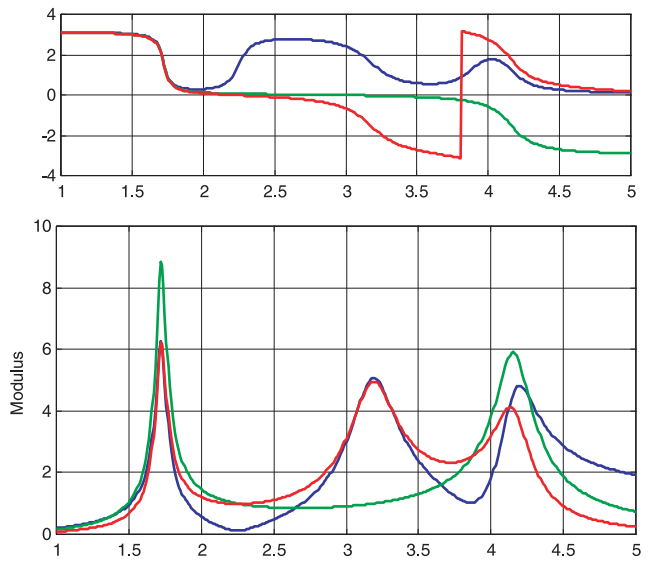

Amplitude/phase

Fig. 3. Exemple de transferts (système à 3 DDL).

- Pour chaque pic de partie imaginaire, détermination de $f_{k}, \zeta_{k}$ et $\tilde{\mathbf{X}}_{a e, k}$ par moindres carrés (identification «à 1 DD $\bar{D}$ »).

- Sélection d'une seule valeur pour $f_{\underline{k}}$ et $\zeta_{\underline{k}}$ de chaque mode $\underline{k}$ (critères possibles : moyenne, valeur minimale de $\zeta_{k}$ souvent sous-estimée à cause de la présence de plusieurs modes dans un seul pic ...).

- Prise en compte des autres modes par résolution d'un système linéaire pour une meilleure approximation des $\tilde{\mathbf{X}}_{a \underline{e}, \underline{k}}$ (identification «multi DDL » mais avec $f_{\underline{k}}$ et $\zeta_{\underline{k}}$ donnés). Comparaison entre transferts mesurés et synthétisés. Itérations possibles (tous les $\tilde{\mathbf{X}}_{\underline{e e}, \underline{k}}$ doivent être strictement positifs - dans le cas contraire : modification de $f_{k}$ et/ou $\zeta_{k}$, avec possibilité de calculs de sensibilité).

- Extraction des paramètres usuels $\boldsymbol{\Phi}_{i k}$ et $m_{\underline{k}}$.

- Identification des termes $\mathbf{X}_{a e, s t}$ et $\overline{\mathbf{X}}_{a e, \text { in }} \frac{\bar{d}^{\prime}}{\text { 'après les }}$ parties réelles aux basses fréquences par moindres carrés suivant $\mathbf{X}_{a \underline{e}, \mathrm{st}}-\mathbf{X}_{a \underline{e}, \text { in }} / \omega^{2}$. Vérification possible des modes rigides correspondants si la géométrie est disponible.

- Comparaison finale entre transferts mesurés et synthétisés, pour une estimation globale de la qualité de l'identification.

Cette démarche doit être précédée d'une étape de récupération et de vérification des données expérimentales. Elle peut être suivie d'un recalage, préalablement préparé, de modèle éléments finis d'après les paramètres identifiés, de manière à conforter les consignes d'essai déduites de ce dernier. La durée totale, estimée à une demi-journée dans de bonnes conditions, est acceptable dans le contexte d'une campagne de qualification.

\section{Illustration}

Dans le cadre de l'étude [2] pour l'ASE, ont été réalisées une simulation numérique avec un modèle par éléments finis du satellite Eurostar 3000 STM (Fig. 4) pour une excitation axiale (verticale), puis une analyse réelle en situation de campagne de qualification du spécimen.

Pour la simulation numérique, un ensemble de transferts définis sur 500 points logarithmiques de 5 à $100 \mathrm{~Hz}$ a été généré à partir du modèle par superposition de 97 modes. L'amplification à résonance $1 /\left(2 \zeta_{k}\right)$ de ces modes a été prise égale à 15 pour les masses effectives importantes et 20 pour les autres, d'où un ensemble de $63(21 \times 3)$ réponses accélérométriques et 6 réactions à la base ( 3 forces et 3 moments) correspondant au dispositif FMD (Force Measurement Device) constitué de 8 cellules de forces et disponible au niveau européen pour ce type d'interface satellite [3].

Les transferts obtenus sont tracés en amplitude sur la figure 5. On peut y distinguer :

- les transferts en accélération (accélération/ accélération = transmissibilité dynamique, sans dimension) dans la direction de l'excitation, qui tendent vers 1 aux basses fréquences,

- les autres transferts en accélération, qui ont une pente en $\omega^{2}$ aux basses fréquences,

- le transfert en réaction (réaction/accélération = masse dynamique, en $\mathrm{kg}$ ou m.kg) dans la direction de l'excitation, donc le transfert ponctuel, qui tend vers la masse statique du spécimen aux basses fréquences, ici $4800 \mathrm{~kg}$,

- les autres transferts en réaction qui tendent vers le terme statique aux basses fréquences (pente en $\omega^{2}$ s'il est nul). 

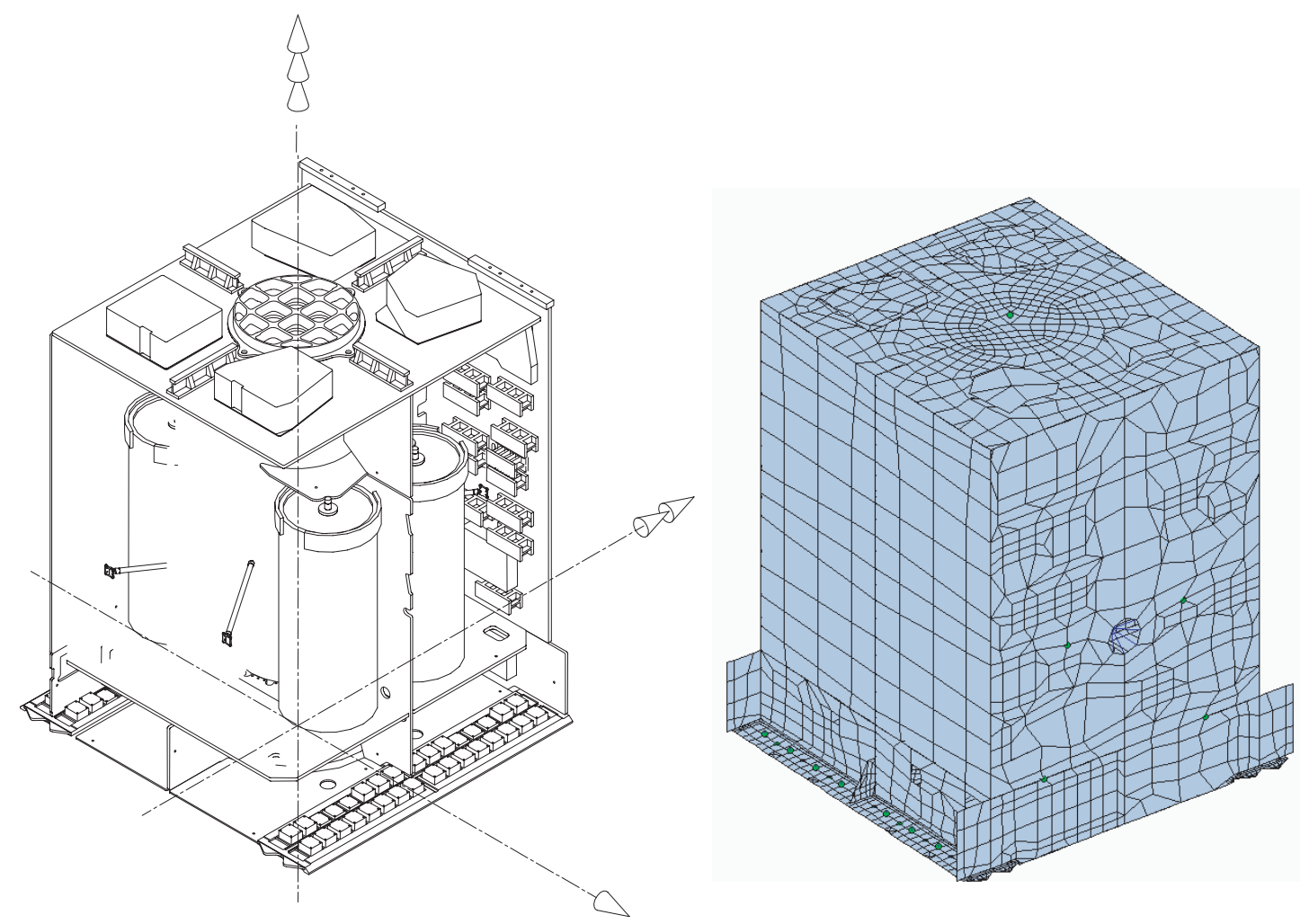

Fig. 4. Eurostar 3000 STM : structure et modèle éléments finis (Astrium/Toulouse).

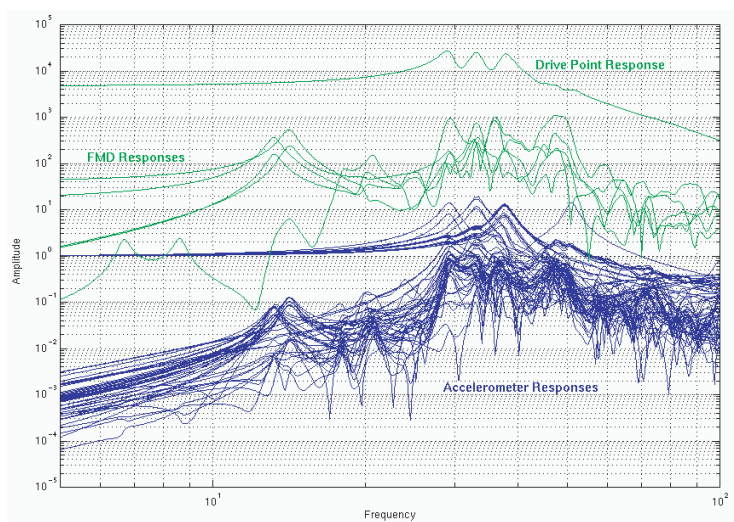

Fig. 5. Transferts expérimentaux simulés.

L'identification modale sur ces transferts a été réalisée avec le logiciel PROTO-Dynamique [4] suivant la procédure de la section 3. L'interface graphique est donnée par la figure 6 avec la partie imaginaire des 69 transferts en exemple.

Le choix des modes propres a été fait en examinant d'abord les 6 masses dynamiques : elles reflètent le comportement du spécimen vu de sa base et jouent donc un rôle fondamental dans le couplage avec le monde extérieur, c'est-à-dire le lanceur pour un satellite. Le transfert ponctuel, en particulier, révèle la présence de 3 modes axiaux importants dans la bande $30-40 \mathrm{~Hz}$ et de 2 modes secondaires vers $10-15 \mathrm{~Hz}$ (faible composante

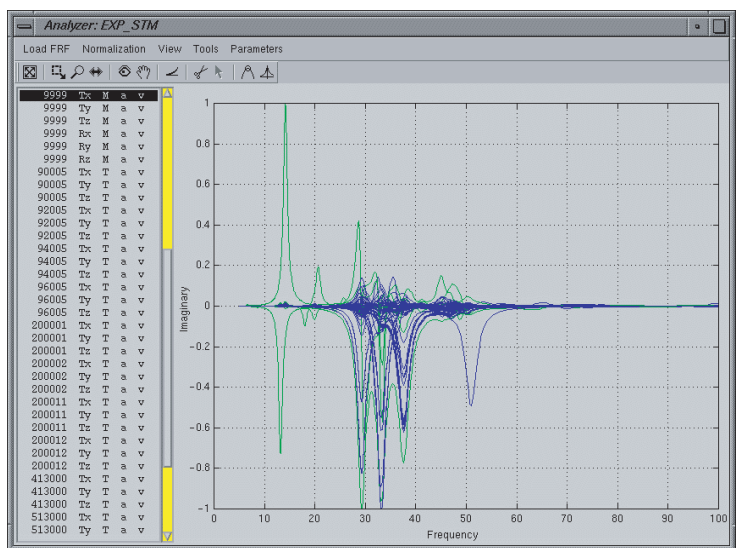

Fig. 6. Module d'identification modale.

axiale des premiers modes latéraux) illustrés par la figure 7.

Pour compléter cette première sélection avec des modes moins importants en masse, mais intéressant des zones sensibles à divers points de vue, l'examen des courbes accélérométriques a abouti au choix des 3 modes supplémentaires de la figure 8 : les deux premiers concernent plusieurs capteurs, alors que le troisième est localisé sur une seule composante.

L'identification de ces 8 modes a donné les résultats de la figure 9 où l'on voit bien l'importance en masse des modes 3 à 5 qui captent à eux seuls $90 \%$ de la masse totale : le mode 3 correspond aux deux réservoirs NTO, 


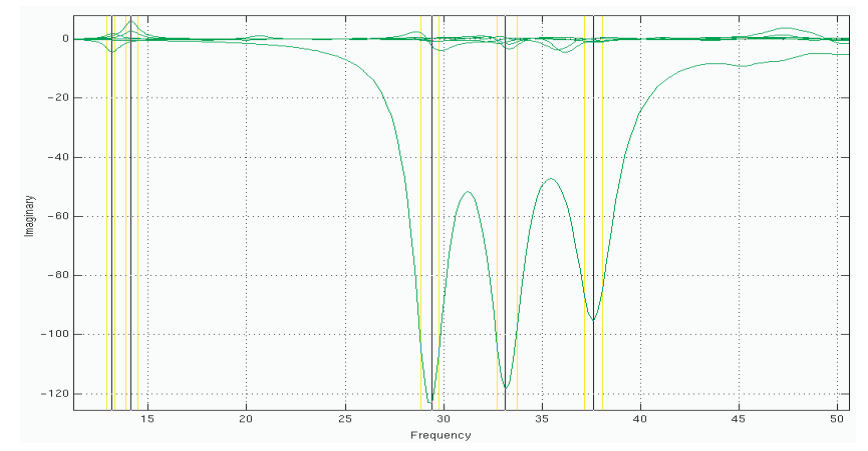

Fig. 7. Sélection de modes « réactions ».

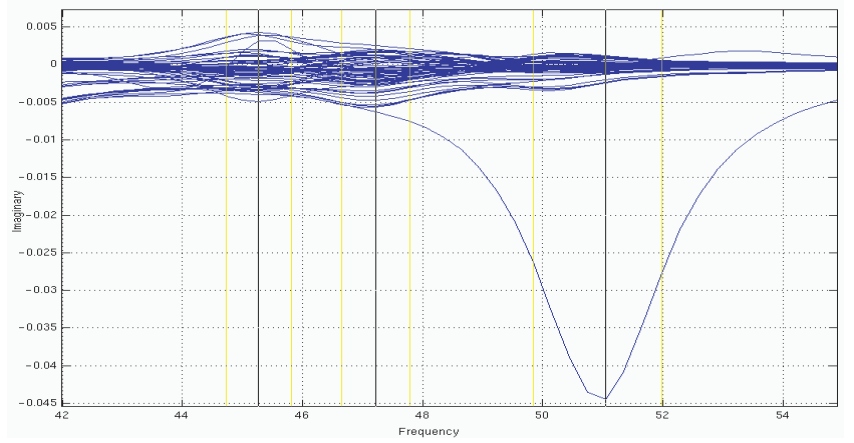

Fig. 8. Sélection de modes « accéléromètres ».

\begin{tabular}{|c|c|c|c|}
\hline Mode & $\begin{array}{l}\text { Freq. } \\
(\mathrm{Hz})\end{array}$ & $\begin{array}{c}\text { Damping } \\
\text { (Q) }\end{array}$ & $\begin{array}{c}\text { Mass } \\
(\mathrm{kg})\end{array}$ \\
\hline 1 & 13.144 & 15.0 & 0.015 \\
\hline 2 & 14.126 & 14.9 & 0.058 \\
\hline 3 & 29.384 & 14.6 & 1438.27 \\
\hline 4 & 33.133 & 15.1 & 1432.24 \\
\hline 5 & 37.585 & 14.7 & 1385.46 \\
\hline 6 & 45.273 & 16.9 & 61.89 \\
\hline 7 & 47.216 & 18.2 & 41.84 \\
\hline 8 & 51.049 & 21.0 & 41.86 \\
\hline
\end{tabular}

Fig. 9. Modes identifiés.

le mode 4 aux deux réservoirs MMH et le mode 5 au module de communications qui constitue la partie supérieure, ces différents éléments structuraux étant visibles sur la figure 4.

D'autres modes identifiables auraient pu être considérés ici mais, d'une part, cette sélection était suffisante pour démontrer la faisabilité de l'approche et, d'autre part, tous les modes n'ont pas la même importance dans ce contexte. Le principal objectif de la campagne d'essai étant la qualification du spécimen, seuls ont un intérêt les modes qui sont susceptibles d'influencer les consignes de réduction de niveau (notching) indispensables pour ces essais de type sinus balayé. En effet, pour un satellite, les spécifications habituelles sont données en terme d'accélération à la base, résultant d'une enveloppe standard des niveaux possibles en vol et ne tiennent donc pas compte du comportement du spécimen en particulier
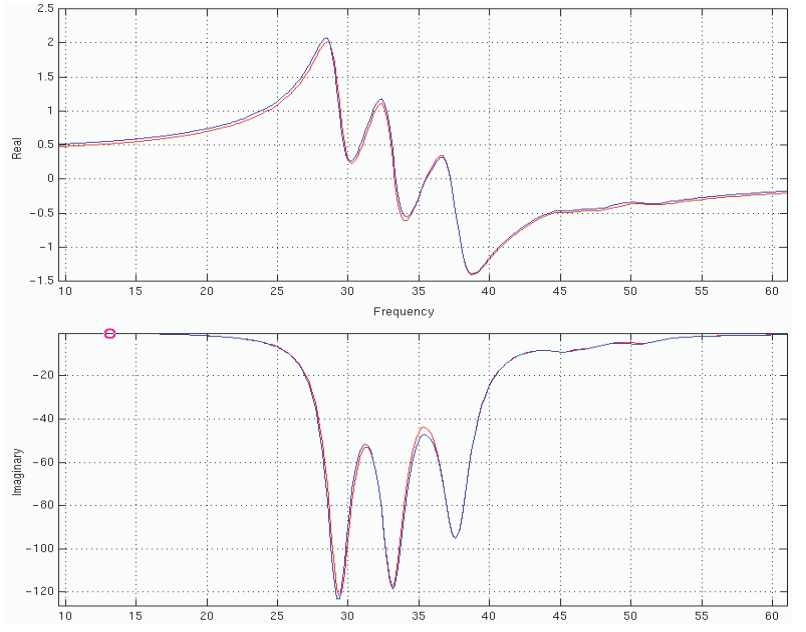

Fig. 10. Exemple de comparaison entre transfert mesuré (simulé) et synthétisé.

à ses propres résonances. Ces dernières doivent donc être réduites, sous certaines réserves, pour avoir des niveaux plus réalistes vis-à-vis du comportement réel en vol, d'où une sélection judicieuse des modes à identifier à cette fin, ce qui a été fait ici.

Un exemple de comparaison entre transfert mesuré et synthétisé est donné par la figure 10, ce qui permet de vérifier la qualité de l'identification pour cette simulation.

L'application réelle a été faite sur le satellite Eurostar 3000 STM dans des conditions analogues à celles d'une campagne de qualification. Une telle campagne comporte en général pour chaque axe un bas niveau qui permet de vérifier la qualité des mesures et le comportement du spécimen, et de déterminer les consignes de réduction de niveau à appliquer pour les forts niveaux. C'est sur ce premier bas niveau que peut se greffer l'identification modale si le temps qu'elle nécessite ne pénalise pas le déroulement normal des opérations. C'est ce qui a été vérifié ici avec un bas niveau axial sur le Moyen de Vibration Système (MVS) d'INTESPACE, utilisant une grande partie des accéléromètres piézoélectriques et des jauges de contrainte déjà collés sur le spécimen, au total plus de 200 voies de mesure, incluant celles relatives au dispositif FMD de mesure des réactions à la base (acquisition par calculateur MASSCOMP et traitement par le logiciel DynaWorks ${ }^{\circledR}$ développé par INTESPACE).

L'examen rapide des réponses a permis de déceler quelques erreurs d'instrumentation. Les modes importants pour les consignes d'essai ont été détectés et convenablement identifiés malgré un niveau de bruit important. Cette étape d'identification modale, en fournissant rapidement des informations peut-être moins précises que les méthodes habituelles, mais suffisantes, a été jugée bien adaptée au contexte. Les principales limitations sont liées au caractère incomplet des données d'essai, à la souplesse du montage et à la présence plus ou moins marquée de bruit de mesure et de mouvements parasites [5]. La durée totale, incluant la corrélation et le recalage de modèle, a été de l'ordre de $4 \mathrm{~h}$, ce qui est comparable au temps 
habituellement utilisé pour préparer un essai à forts niveaux et donc compatible avec le déroulement normal d'une campagne de qualification.

\section{Conclusion}

L'identification modale via les paramètres effectifs peut être utilisée en cas d'excitation par force ou par la base. Pour ce type d'identification, un outil interactif, robuste et efficace en présence d'incertitudes de mesure a été élaboré, incluant la détection/sélection des modes d'après visualisation graphique, la vérification des données et l'extraction des paramètres modaux et statiques à partir des parties réelles/imaginaires des transferts. Cet outil permet en particulier l'identification modale d'un satellite en campagne de qualification en fournissant rapidement des informations entre deux essais à des fins de vérification.

Remerciements. Ces travaux ont été réalisés grâce à l'ASE/ESTEC (A. Calvi) qui a supporté en partie la recherche dans le cadre de l'étude [2] et Astrium/Toulouse
(Y.-M. Lefèvre entre autres) qui a fourni le modèle de simulation numérique et fait l'exploitation relative à la campagne d'essais. Ils ont été menés à bien avec N. Roy maintenant gérant de la société Top Modal.

\section{Références}

[1] A. Girard, N. Roy, Dynamique des structures industrielles, Hermes-Lavoisier, 2003

[2] Real time modal vibration identification for communication satellite testing, ESTEC Invitation to Tender AO3458, March 1999

[3] Force measurement device (FMD) dynamic validation, Final Report INGEMANSSON V-1211-B 28/01/98

[4] PROTO-Dynamique User's Manual Version 4, INTESPACE Document DO 99.013 ER, June 23, 1999

[5] Y.-M. Lefèvre, J.-C. Bonetti, A. Girard, N. Roy, A. Calvi, Real-time modal identification techniques for improved satellite vibration testing, European Conference on Spacecraft Structures, Materials and Mechanical Testing, Toulouse, 11-13 December 2002 